\title{
Dispelling dog dogma: an investigation of heterochrony in dogs using 3D geometric morphometric analysis of skull shape
}

\author{
Abby Grace Drake* \\ Department of Biology, College of the Holy Cross, P.O. Box B 1 College Street Worcester, MA 01610, USA \\ *Author for correspondence (email: abbygracedrake@googlemail.com)
}

SUMMARY Heterochrony is an evolutionary mechanism that generates diversity via perturbations of the rate or timing of development that requires very little genetic innovation. As such, heterochrony is thought to be a common evolutionary mechanism in the generation of diversity. Previous research has suggested that dogs evolved via heterochrony and are paedomorphic wolves. This study uses three-dimensional landmark-based coordinate data to investigate heterochronic patterns within the skull morphology of the domestic dog. A total of 677 adult dogs representing 106 different breeds were measured and compared with an ontogenetic series of 401 wolves. Geometric morphometric analysis reveals that the cranial shape of none of the modern breeds of dogs resembles the cranial shapes of adult or juvenile wolves. In addition, investigations of regional heterochrony in the face and neurocranium also reject the hypothesis of heterochrony. Throughout wolf cranial development the position of the face and the neurocranium remain in the same plane. Dogs, however, have a de novo cranial flexion in which the palate is tilted dorsally in brachycephalic and mesaticephalic breeds or tilted ventrally in dolichocephalic and down-face breeds. Dogs have evolved very rapidly into an incredibly morphologically diverse species with very little genetic variation. However, the genetic alterations to dog cranial development that have produced this vast range of phylogenetically novel skull shapes do not coincide with the expectations of the heterochronic model. Dogs are not paedomorphic wolves.

\section{INTRODUCTION}

The vast morphological diversity of the domestic dog (Canis lupus familiaris) has arisen relatively rapidly and with little genetic modification (Wayne 1986; Drake and Klingenberg 2010). Heterochrony is an evolutionary process thought to be able to generate diversity via changes in the rate or timing of ontogenetic pathways (Gould 1977) which would require only simple genetic modifications and has specifically been suggested as the mechanism responsible for the evolution of the domestic dog (Bolk 1926; Dechambre 1949; Howard 1949; Zeuner 1963; Fox 1965; Lawrence and Bossert 1969; CluttonBrock et al. 1976; Olsen and Olsen 1977; Fox 1978; Coppinger and Coppinger 1982; Frank and Frank 1982; Price 1984; Wayne 1986; Coppinger et al. 1987; Morey 1992; Morey 1994; Coppinger and Coppinger 2001). In fact, some suggest that paedomorphosis, the retention of juvenile features in sexually mature adults, is the most common evolutionary process in animal domestication (Price 1984; McKinney 1999).

Three components, which may act independently or in concert, comprise an organism's ontogeny: growth, development, and maturation (Gould 1977). Growth is the geometric increase in size of an organism while development is the modification of an organism's shape. Heterochrony operates by perturbing any of these components of the ancestral ontogenetic trajectory to produce a descendant that either resembles the ancestor at a younger stage of development (paedomorphosis) or at a point of continued development (peramorphosis), is smaller or larger, or some combination thereof. Growth and development overlap in the realm of allometry. Allometry occurs as proportional changes in shape that are directly related to changes in size. Most researchers only measure heterochronic disturbances to development that result in allometric differences because of the historical precedent set by Gould (1977) and later by Alberch et al. (1979) in relating development directly to allometry. Allometry is traditionally measured by growth curves and bivariate plots, which provide useful metrics for measuring shape change. However, as developmental biologists have shown, many of the complex aspects of biological development are independent of size (Raff and Wray 1989). Although heterochrony is often seen as a purely allometric model it is possible that parallels in nonallometric variation may occur through common differentiation patterns.

Geometric morphometrics is a powerful multivariate tool that allows for both separate and simultaneous analysis of different ontogenetic modifications (Mitteroecker et al. 2004, 2005). The first step in geometric morphometric analysis is the 
Procrustes superimposition which scales specimen coordinate configurations to the same size (and also removes variation due to rotation and translation) such that size (centroid size [CS], Dryden and Mardia 1998) is separated from shape. Further analysis allows exploration of the most important aspects of shape variation via a re-ordination in the form of principal components analysis (PCA). In addition, those aspects of shape variation that are purely allometric can be examined by a multivariate regression of the Procrustes shape coordinates on CS. In turn, the nonallometric component of shape variation may be investigated by a PCA of the residuals from the multivariate regression. These multivariate analyses of shape variation and its association with or independence from size variation are inherent to any study of heterochrony.

Gould (1977) limited heterochronic evolution to only those modifications in the relative timing of developmental events that result in parallels between ontogeny and phylogeny. If not restricted to these parallels between ancestral and descendent ontogenies, then heterochrony fails to quantify only shared developmental trajectories, and thus every developmental change would fall under the rubric of heterochrony. de Beer described paedomorphim as "A character which is present or makes its appearance in the young stage of an ancestral animal may in the ontogeny of the descendant appear in the adult ..." (de Beer 1930, p. 37). Gould's clock models were formulated in order that the adult descendant size and shape may be directly compared with the ancestral ontogenetic trajectory (Gould 1977). Therefore, in testing for paedomorphism in dogs we can compare the shapes of adult dog crania to the range of shape changes in the wolf ontogeny to investigate similarities (Klingenberg 1998). Blanco and Godfrey (2005) conducted a similar test for ontogenetic scaling as a mechanism for generating sexual dimorphism in howler monkeys by comparing the shapes of adult female howler monkeys to similarly sized subadult males. They found that the cranial shape of adult female monkeys did not resemble the cranial shape of the subadult males in multivariate analyses. Evolution via heterochronic processes can be detected by direct comparison of adult descendant morphologies to the sequence of shapes found on the ancestral developmental trajectory. If similarities in shape are found between the adult descendant and the ancestral ontogeny then further tests to show that they share the same ontogentic pathway are needed to ensure a diagnosis of heterochrony (Godfrey et al. 1998). However, if no shape similarity is found then heterochrony can be rejected without further tests.

How useful is heterochrony as a model of evolutionary change? Is paedomorphism prevalent in dogs? Superficial appearances and certain care-soliciting behaviors have led to the speculation that dogs are paedomorphic. Dogs were first proposed to be heterochronically derived by Bolk as further support for his controversial heterochronic classification of humans (Bolk 1926; Gould 1977). As a result several re- searchers have conducted tests of the heterochrony hypothesis in dogs using cranial morphology (Dechambre 1949; Wayne 1986; Morey 1992). In the most significant paper on the morphological evolution of dogs to date, Wayne (1986) compared adult dog crania from a variety of breeds to those of wild canid species and to an ontogenetic series of dogs (German Shepherds) on bivariate $\log -\log$ plots and found that most small breeds were paedomorphic for some cranial ratios, especially the width of the skull. However, it has been pointed out that it is difficult to reject a hypothesis of heterochrony on bivariate plots because every descendant will have a shape ratio that intersects with the ancestral ontogenetic trajectory, albeit at different sizes (for a discussion of this see: Zelditch and Fink 1996; Mitteroecker et al. 2005).

This study reanalyzes the heterochrony hypothesis in dogs using three-dimensional coordinates from 64 cranial landmarks taken from an ontogenetic series of wolves and a large sample of adult dogs that represents their entire phenotypic spectrum. Multivariate geometric morphometric techniques which facilitate holistic analysis of trait covariation (Mitteroecker et al. 2005) are used to test for similarities in cranial shape that may have been produced by heterochronic processes. This study examines allometric shape variation as well as size-independent nonallometric shape variation in order to capture all possible developmental changes during the evolution of the domestic dog. In addition, separate analyses are conducted for the facial region and the neurocranium which have been shown to be developmentally modular in dogs and wolves (Drake and Klingenberg 2010).

\section{MATERIALS AND METHODS}

\section{Data set}

A MicroScribe digitizer was used to capture the three-dimensional coordinates of 64 cranial landmarks (Fig. 1) on the dorsal and ventral aspects of 401 skulls for an ontogenetic series of wolves, Canis lupus lupus and 677 skulls of adult dogs, C. l. familiaris, representing 106 breeds (see supporting information Table 1). The dorsal and ventral configurations were combined into a set of coordinates for the whole skull using a least-squares fit (rotation and translation only) of four matching landmarks that were digitized on both halves. Every skull was digitized twice in order to account for measurement error. Specimens are housed at the Smithsonian Institution's National Museum of Natural History in Washington, DC, the private collection of Bonnie Dazell, the University of Alaska Museum in Fairbanks, Alaska, the Museum of Vertebrate Zoology at the University of California in Berkeley, California, the Natural History Museum, Zoology Department, London, and the Natural History Museum in Berne, Switzerland.

Dental eruption pattern was used to classify the ontogenetic series of wolves into three developmental stages (Miller 1964). Newborn wolves, without any erupted teeth, through juvenile wolves, with all deciduous teeth erupted, were placed in the "Deciduous" group $(N=32)$. Wolves whose permanent teeth had 


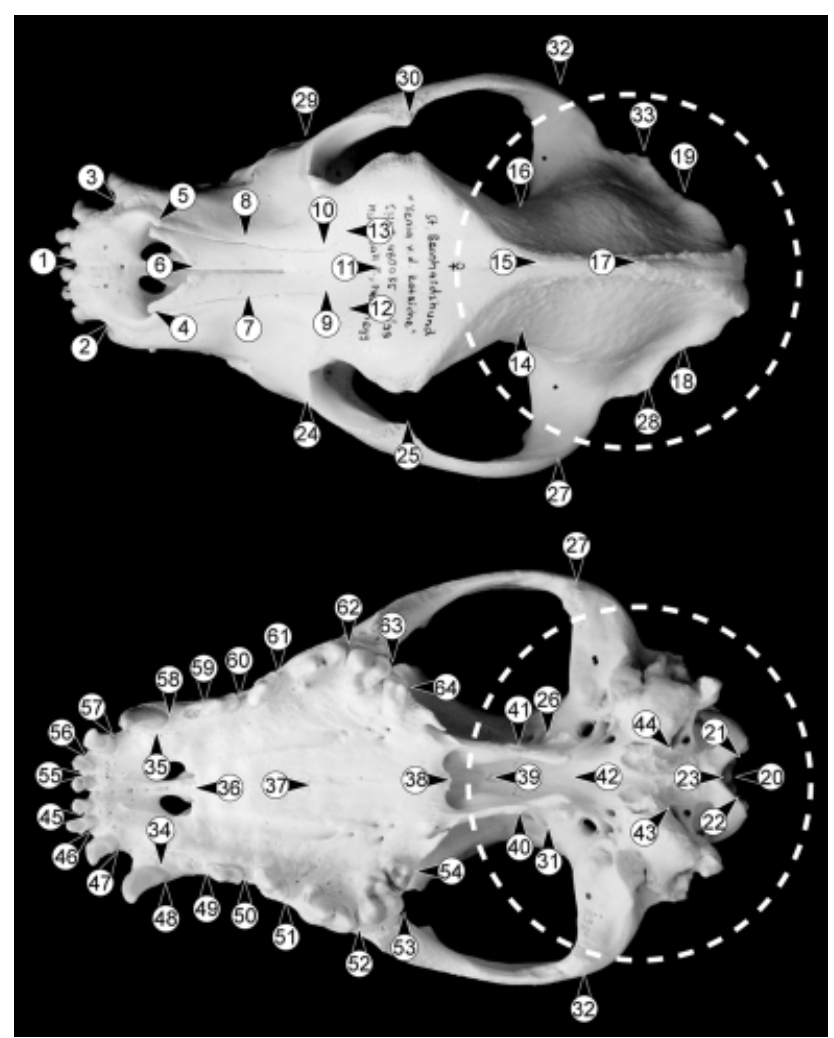

Fig. 1. The 64 ostoleogical landmarks used for morphometric analysis.

begun to emerge through those with the complete loss of all deciduous teeth were placed in the "Permanent" group $(N=45)$. Wolves with all permanent teeth fully erupted were placed in the "Adult" group $(N=324)$.

\section{Shape analysis}

A geometric morphometric analysis of the $3 \mathrm{D}$ coordinate data was performed with MorphoJ software (Klingenberg 2011). Generalized least-squares Procrustes superimposition was used to extract geometric shape variation for all dogs and the ontogenetic series of wolves simultaneously. Procrustes superimposition removes all information that is related to scaling, position or rotation (Dryden and Mardia 1998), yielding Procrustes shape coordinates that were used in subsequent analyses. Procrustes shape coordinates do not contain any size variation due to scaling or isometric variation. However, Procrustes shape coordinates do include allometric shape variation - that is shape variation that is directly related to size variation. CS, the sum of the squared distances from each landmark to the centroid, was calculated for each configuration.

There was an insignificant amount of measurement error among replicated digitizations as calculated using Procrustes ANOVA (Klingenberg et al. 2002); allowing all replicates to be averaged into a single configuration for each specimen.

PCA based on the covariance matrix of the Procrustes shape coordinates of all dogs and wolves was used to explore patterns of shape variation. Hotelling's $T^{2}$ was used to test for significant differences in principal component scores between groups.
The nonallometric shape component was separated from the Procrustes coordinate data by a multivariate pooled within-group linear regression of the Procrustes shape coordinates against log CS. The residuals from this regression contain the nonallometric component of shape variation and were used to analyze non-sizerelated shape variation.

In order to quantify differences in shape, calculations of Mahalanobis and Procrustes distances were performed with 10,000 permutations per test to estimate significance of between group differences for both the allometric and nonallometric components of shape variation (Good 2000). While Procrustes distance is a measure of the absolute distance between group means, Mahalanobis distance takes into account the within-group variation when calculating between group mean distances (Klingenberg and Monteiro 2005). There is some debate over which distance test is more appropriate so both are included in this study along with Hotelling's $T^{2}$-test for differences between group mean PC scores (Klingenberg and Monteiro 2005). There are no discrepancies in the results of the tests as all find significant differences in each application. The Landmark software was used to morph a 3D digital scan of an adult wolf skull in order to visualize shape variation along the PC axes using a thin plate spline interpolation function (Bookstein 1991; Wiley et al. 2005).

Drake and Klingenberg (2010) found evidence for phenotypic modularity of the face and neurocranium in both dogs and wolves, and importantly they found developmental modularity of these two regions as well. Many researchers (Gould 2002; Mitteroecker et al. 2004, 2005) have pointed out that it may be unrealistic to expect "pure heterochrony," that morphological elements of entire organisms evolve as integrated wholes by the same heterochronic process, and they therefore encouraged the investigation of heterochrony within suites of traits. In the present study, heterochrony is investigated for the whole cranium and as well as the neurocranium (19 landmarks, within the dashed circle on Fig. 1) and the face (45 landmarks).

\section{RESULTS}

It has previously been shown that most breeds of dogs have phylogenetically novel skull shapes when compared with adult wolves (Drake and Klingenberg 2010). This dissimilarity in cranial shape is further confirmed by the significant differences found for both Mahalanobis and Procrustes distance tests between all of the adult dog cranial shapes and all stages of wolf cranial development (Table 1a).

Other studies have shown that a significant portion of the shape variation within dogs is allometric (Wayne 1986; Drake and Klingenberg 2010). A multivariate regression of the entire set of Procrustes coordinates for all wolves and dogs against CS shows that allometry alone explains $33.8 \%$ of the skull shape variation and is statistically significant $(P<0.0001)$. Using the residuals from the multivariate regression to investigate the nonallometric component of shape variation, all of the permutation tests for both the Procrustes and 
Table 1. Cranial shape differences

\begin{tabular}{|c|c|c|c|c|}
\hline & Mahalanobis distance & $P$-value & Procrustes distance & $P$-value \\
\hline \multicolumn{5}{|l|}{ (a) Procrustes shape variation } \\
\hline All wolves (402)—all dogs (683) & 7.19 & $<0.0001$ & 0.06 & $<0.0001$ \\
\hline Adult wolves (324) —all dogs (683) & 7.46 & $<0.0001$ & 0.07 & $<0.0001$ \\
\hline Permanent wolves (45) - all dogs (683) & 8.31 & $<0.0001$ & 0.06 & $<0.0001$ \\
\hline Deciduous wolves (32) — all dogs (683) & 12.32 & $<0.0001$ & 0.10 & $<0.0001$ \\
\hline All wolves (402)—mesaticephalic dogs (259) & 8.82 & $<0.0001$ & 0.04 & $<0.0001$ \\
\hline Adult wolves (324) — mesaticephalic dogs (259) & 9.03 & $<0.0001$ & 0.04 & $<0.0001$ \\
\hline Permanent wolves (45) —mesaticephalic dogs (259) & 10.06 & $<0.0001$ & 0.04 & $<0.0001$ \\
\hline Deciduous wolves (32) — mesaticephalic dogs (259) & 14.88 & $<0.0001$ & 0.14 & $<0.0001$ \\
\hline \multicolumn{5}{|l|}{ (b) Nonallometric shape variation } \\
\hline All wolves (402) — all dogs (683) & 4.22 & $<0.0001$ & 0.04 & $<0.0001$ \\
\hline Adult wolves (324) —all dogs (683) & 4.51 & $<0.0001$ & 0.04 & $<0.0001$ \\
\hline Permanent wolves (45) - all dogs (683) & 7.13 & $<0.0001$ & 0.05 & $<0.0001$ \\
\hline Deciduous wolves (32) — all dogs (683) & 12.27 & $<0.0001$ & 0.07 & $<0.0001$ \\
\hline All wolves (402) - mesaticephalic dogs & 6.03 & $<0.0001$ & 0.04 & $<0.0001$ \\
\hline Adult wolves (324) —mesaticephalic dogs & 7.8 & $<0.0001$ & 0.04 & $<0.0001$ \\
\hline Permanent wolves (45) - mesaticephalic dogs & 9.97 & $<0.0001$ & 0.04 & $<0.0001$ \\
\hline Deciduous wolves (32) - mesaticephalic dogs & 13.73 & $<0.0001$ & 0.07 & $<0.0001$ \\
\hline
\end{tabular}

Mahalanobis distances between each of the wolf developmental stages and the dogs once again confirmed significant differences in cranial shape (Table 1b).

Much of the variation among the dogs is due to the extreme disparity of the brachycephalic (short, broad skulls), doliocephalic (elongate, narrow skulls), and down-face (muzzle is angled down) breeds. Therefore, a separate analysis limited to only mesaticephalic (skulls of average shape) breeds, such as German Shepherds and Labradors, was conducted. Even when the dog sample was limited to only mesaticephalic breeds, there was still a significant difference between dog cranial shape and all stages of wolf cranial development for both the Procrustes shape coordinates and the multivariate regression residuals (Table 1). These results demonstrate that adult dogs do not share the same allometric or nonallometric craniofacial shape space as the wolf developmental trajectory.

PCA allows for an exploratory analysis of the dataset where a priori group structure does not influence the outcome. A PCA of the Procrustes shape coordinates illustrates that many dogs have cranial shapes that are distinct from either adult or juvenile wolves (Fig. 2A) (Lawley-Hotelling PCs $\left.1-3, T_{(9,3224)}=0.87, P<0.0001\right)$. The first three PCs account for $75.3 \%$ of the total variance $(\mathrm{PCl}=59.5 \%$, $\mathrm{PC} 2=10.5 \%$, and $\mathrm{PC} 3=5.3 \%$ ). When the variation within the dog sample was reduced to the average for each breed, there were still significant differences between the cranial shape variation of the ontogentic series of wolves and the dogs (Lawley-Hotelling PCs $1-3, T_{(9,1511)}=2.02, P<0.0001$ ). This PCA captures the extreme neomorphic variation of the brachycephalic, dolichocephalic, and down-face breeds
(Fig. 2A). Principal component one shows the variation from short, wide, and round skulls to those with more elongate, narrow crania; PC2 contrasts the pronounced stop of the brachycephalic breeds with the relatively flat muzzles of the wolves and dolichocephalic dogs; and PC3 illustrates the extreme down-faced muzzle of the Bull Terrier. Though most of the mesaticephalic dogs coincide with the wolf series, a few are separated from the wolves. A separate PCA comparing only the mesaticephalic dogs to the ontogenetic series is conducted later in this article.

The residuals from the multivariate regression of the Procrustes shape coordinates versus CS were used to compute the covariance matrix for a PCA of the nonallometric component of shape variation (Fig. 2B). Most dogs are separated from wolves in the nonallometric shape space of the first three PCs (Lawley-Hotelling PCs 1-3, $\left.T_{(3,3244)}=1.11, P<0.0001\right)$ $(\mathrm{PC} 1=50.7 \%, \mathrm{PC} 2=8.6 \%$, and $\mathrm{PC} 3=5.0 \%)$. The nonallometric differences between the ontogenetic series of wolves and dogs is not only due to variation within breeds, the position of most breed means are also outside the variation of head shape of the ontogenetic series of wolves (LawleyHotelling PCs $\left.1-3, T_{(9,1511)}=1.58, P<0.0001\right)$. Principal component one contrasts the brachycephalic breeds with the doliocephalic breeds both of which are outside the range of wolf cranial shapes. The cranial shape variation along PC1 ranges from the brachycephalic wide, round skulls, and dorsally tilted palates to the elongate, narrow, and klinorhynchic dolichocephalic skulls. This nonallometric component of the cranial shape variation among dogs demonstrates that some of the phylogenetically novel dog skull shapes are not due to simple allometric variation. 

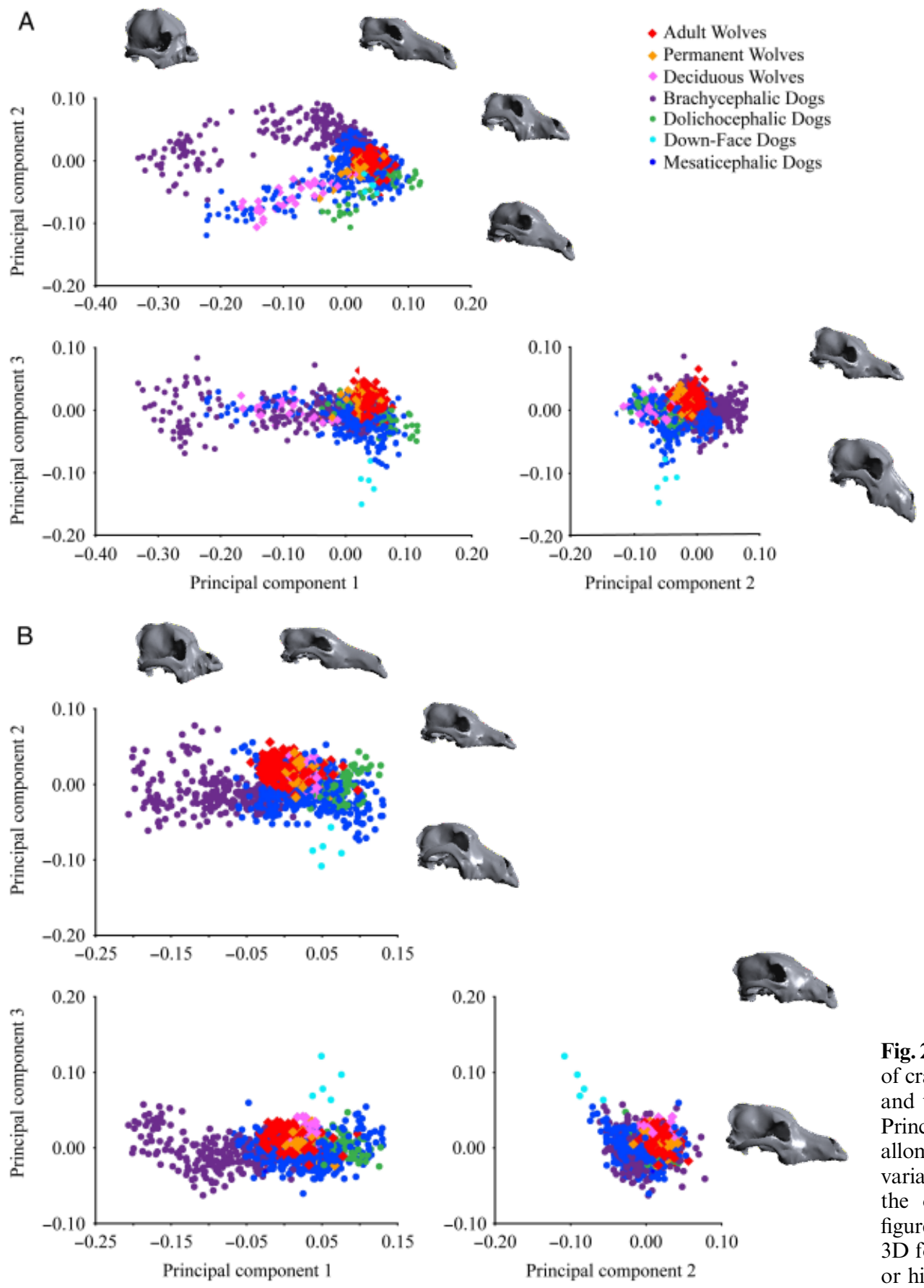

Fig. 2. (A) Principal component analysis of cranial shape comparing all adult dogs and the ontogenetic series of wolves. (B) Principal component analysis of the nonallometric component of cranial shape variation comparing all adult dogs and the ontogenetic series of wolves. This figure is available online in an interactive $3 \mathrm{D}$ format that requires Adobe Reader 9 or higher to view.

\section{Mesaticephalic dogs}

Because most of the variation illustrated in the above PCA is due to the contrast between the highly derived brachycephalic, dolichocephalic, and down-face breeds, a separate analysis including only a subset of the mesaticephalic breeds (those breeds overlapping with the wolves in the above analysis) and the ontogenetic series of wolves was performed. Even this much narrower subsample of dog phenotypes was signifi- cantly different from the ontogenetic series of wolves (Table 1). All of the Procrustes and Mahalanobis distance tests were found to be significant for both the analysis of the Procrustes coordinates and the nonallometric component of shape variation $(P<0.0001)$.

The PCA of the Procrustes coordinates further contrasts the differences between these two groups in shape space. While the first PC explains $35.9 \%$ of the variance and 

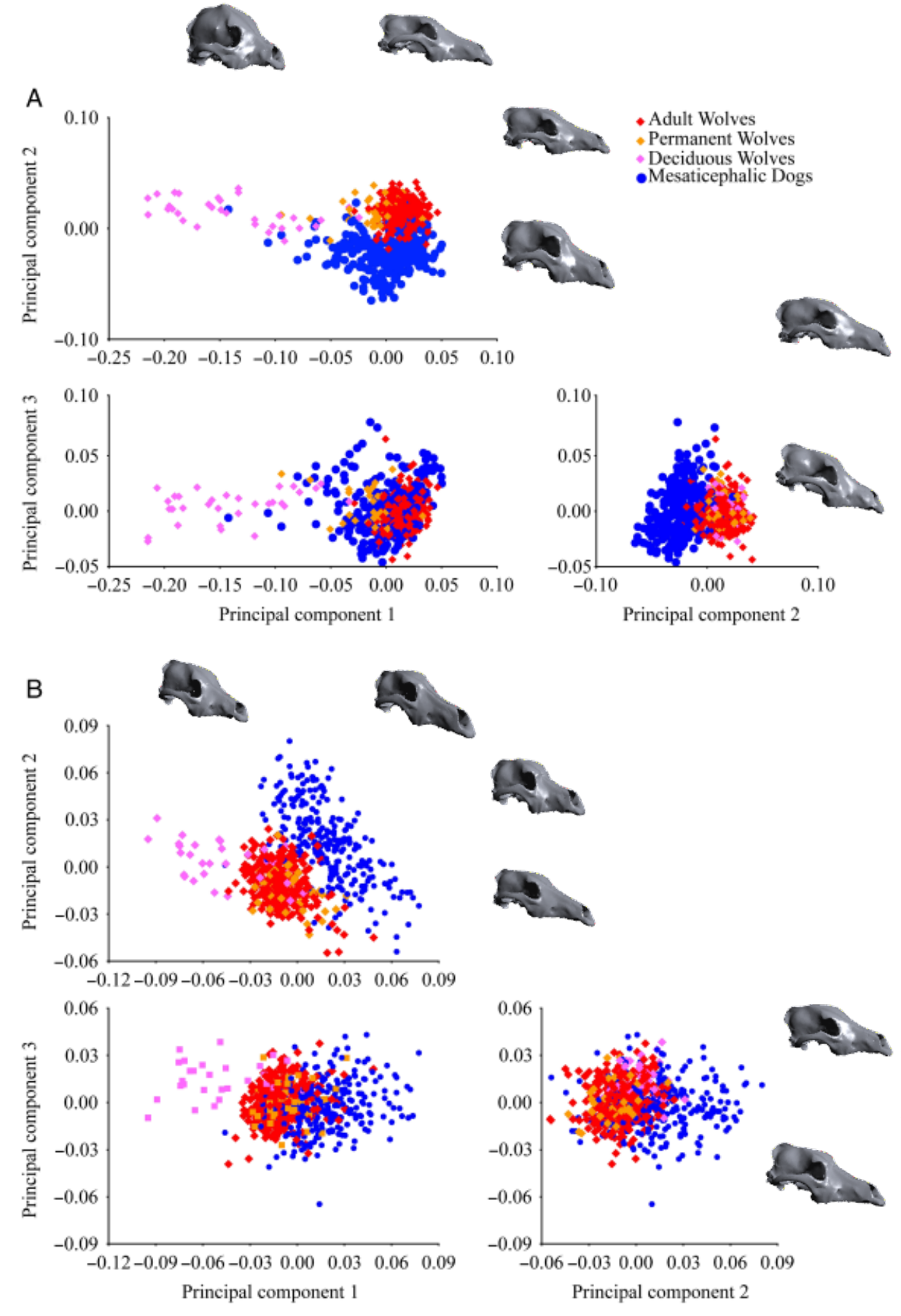

Fig. 3. (A) Principal component analysis of cranial shape comparing mesaticephalic adult dogs and the ontogenetic series of wolves. (B) Principal component analysis of the nonallometric component of cranial shape variation comparing mesaticephalic adult dogs and the ontogenetic series of wolves. This figure is available online in an interactive 3D format that requires Adobe Reader 9 or higher to view.

captures the allometric shape variation in the ontogenetic series of wolves $\left(R^{2}=0.74, P<0.0001\right)$, PC2, which explains $12 \%$ of the total variance, separates the mesaticephalic dogs from the ontogenetic series of wolves (Lawley-Hotelling PCs $\left.1-3, T_{(9,1961)}=4.53, P<0.0001\right)$ (Fig. 3A). Mesaticephalic dogs, which score negatively on PC2 have a more pronounced stop and dorsally tilted palate than the wolf cranial shapes associated with the positive end of PC2
(Fig. 3A). Additionally, in a PCA of the nonallometric component of shape variation the mesaticephalic breeds are separated from the wolves on both PCs $1(18.57 \%)$ and $2(15.5 \%)$ (Lawley-Hotelling PCs $1-3, \quad T_{(9,1961)}=3.24$, $P<0.0001$ ) (Fig. 3B). Both of these results confirm that even this narrow subset of mesaticephalic dog breeds have skull morphologies that are distinguished from adult and juvenile wolves. 
Table 2. Neurocranial shape differences

\begin{tabular}{|c|c|c|c|c|}
\hline & Mahalanobis distance & $P$-value & Procrustes distance & $P$-value \\
\hline \multicolumn{5}{|l|}{ (a) Procrustes shape variation } \\
\hline All wolves (402) — All dogs (683) & 4.08 & $<0.0001$ & 0.09 & $<0.0001$ \\
\hline Adult wolves (324) —all dogs (683) & 4.28 & $<0.0001$ & 0.10 & $<0.0001$ \\
\hline Permanent wolves (45)—all dogs (683) & 4.57 & $<0.0001$ & 0.09 & $<0.0001$ \\
\hline Deciduous wolves (32) - all dogs (683) & 6.26 & $<0.0001$ & 0.11 & $<0.0001$ \\
\hline All wolves (402)—mesaticephlic dogs (259) & 4.16 & $<0.0001$ & 0.07 & $<0.0001$ \\
\hline Adult wolves (324) - mesaticephlic dogs (259) & 4.56 & $<0.0001$ & 0.08 & $<0.0001$ \\
\hline Permanent wolves (45) - mesaticephalic dogs (259) & 4.95 & $<0.0001$ & 0.08 & $<0.0001$ \\
\hline Deciduous wolves (32) - mesaticephalic dogs (259) & 7.08 & $<0.0001$ & 0.14 & $<0.0001$ \\
\hline \multicolumn{5}{|l|}{ (b) Nonallometric shape variation } \\
\hline All wolves (402) — all dogs (683) & 3.18 & $<0.0001$ & 0.07 & $<0.0001$ \\
\hline Adult wolves (324) — all dogs (683) & 3.13 & $<0.0001$ & 0.07 & $<0.0001$ \\
\hline Permanent wolves (45) — all dogs (683) & 4.24 & $<0.0001$ & 0.08 & $<0.0001$ \\
\hline Deciduous wolves (32) —all dogs (683) & 6.21 & $<0.0001$ & 0.09 & $<0.0001$ \\
\hline All wolves (402) - mesaticephlic dogs (259) & 3.66 & $<0.0001$ & 0.07 & $<0.0001$ \\
\hline Adult wolves (324) - mesaticephalic dogs (259) & 3.71 & $<0.0001$ & 0.07 & $<0.0001$ \\
\hline Permanent wolves (45) — mesaticephalic dogs (259) & 4.91 & $<0.0001$ & 0.08 & $<0.0001$ \\
\hline Deciduous wolves (32) - mesaticephalic dogs (259) & 6.19 & $<0.0001$ & 0.08 & $<0.0001$ \\
\hline
\end{tabular}

\section{Regional heterochrony: neurocranium and face}

Significant differences between dogs and adult or juvenile wolves were found for both the neurocranium and the face according to both Procrustes and Mahalanobis distance tests (Tables 2 and 3). A multivariate regression of the Procrustes coordinates on $\log$ CS explained $29.11 \%$ of the shape variation in the neurocranium and $30.05 \%$ of the shape variation in the face. The nonallometric component of shape variation confirmed statistically significant differences between wolves and dogs for both regions as well (Tables 2 and 3). In addition, even the subsample of mesaticephalic breeds was found to significantly differ from the ontogenetic series of wolves in Procrustes shape space and nonallometric shape space for both of the facial and the neurocranial regions (Tables 2 and 3).

Differences in the facial and neurocranial shape of wolves and dogs were further confirmed by MANOVAs of the PC scores for the first three principal components. Both allometric and nonallometric components of shape variation were

Table 3. Facial shape differences

\begin{tabular}{|c|c|c|c|c|}
\hline & Mahalanobis distance & $P$-value & Procrustes distance & $P$-value \\
\hline \multicolumn{5}{|l|}{ (a) Procrustes Shape Variation } \\
\hline All wolves (402) — all dogs (683) & 5.98 & $<0.0001$ & 0.07 & $<0.0001$ \\
\hline Adult wolves (324) — all dogs (683) & 6.31 & $<0.0001$ & 0.08 & $<0.0001$ \\
\hline Permanent wolves (45) —all dogs (683) & 6.82 & $<0.0001$ & 0.08 & $<0.0001$ \\
\hline Deciduous wolves (32) — all dogs (683) & 9.70 & $<0.0001$ & 0.12 & $<0.0001$ \\
\hline All wolves (402)—mesaticephalic dogs (259) & 7.19 & $<0.0001$ & 0.05 & $<0.0001$ \\
\hline Adult wolves (324) - mesaticephalic dogs (259) & 7.48 & $<0.0001$ & 0.05 & $<0.0001$ \\
\hline Permanent wolves (45)—mesaticephalic dogs (259) & 8.30 & $<0.0001$ & 0.06 & $<0.0001$ \\
\hline Deciduous wolves (32) — mesaticephalic Dogs (259) & 10.95 & $<0.0001$ & 0.16 & $<0.0001$ \\
\hline \multicolumn{5}{|l|}{ (b) Nonallometric shape variation } \\
\hline All wolves (402)—all dogs (683) & 3.99 & $<0.0001$ & 0.05 & $<0.0001$ \\
\hline Adult wolves (324) — all dogs (683) & 4.03 & $<0.0001$ & 0.05 & $<0.0001$ \\
\hline Permanent wolves (45) — all dogs (683) & 5.91 & $<0.0001$ & 0.07 & $<0.0001$ \\
\hline Deciduous wolves (32) — all dogs (683) & 9.53 & $<0.0001$ & 0.09 & $<0.0001$ \\
\hline All wolves (402)—mesaticephalic dogs (259) & 5.63 & $<0.0001$ & 0.05 & $<0.0001$ \\
\hline Adult wolves (324) — mesaticephalic dogs (259) & 6.19 & $<0.0001$ & 0.05 & $<0.0001$ \\
\hline Permanent wolves (45) — mesaticephalic dogs (259) & 7.94 & $<0.0001$ & 0.06 & $<0.0001$ \\
\hline Deciduous wolves (32) - mesaticephalic dogs (259) & 9.77 & $<0.0001$ & 0.08 & $<0.0001$ \\
\hline
\end{tabular}


tested for these two regions for all of the dogs as well as the subset of mesaticephalic breeds. In all of the tests significant differences were established $(P<0.0001)$.

\section{Wolf cranial development}

What shape changes take place during the development of the wolf cranium that make the ancestral ontogenetic trajectory so different from all of the various dog skull phenotypes? Using only the ontogenetic series of wolves, an additional PCA on the covariance matrix of the Procrustes coordinates was conducted to illustrate wolf cranial developmental (Fig. 4). The first principal component $(51.7 \%)$ shows that wolf puppies have relatively wide skulls and short muzzles. However, unlike the dogs, the wolf palate and basicranium remain in the same plane throughout development. While much of the variation seen in dogs, especially in the most aberrant breeds, is due to cranial flexion; wolves do not exhibit this shape change at any point during development. Rather, during wolf cranial development the muzzle elongates and the neurocranium narrows.

\section{DISCUSSION}

Dogs are not paedomorphic wolves. Whether brachycephalic, doliocephalic, down-face or even mesaticephalic, all dogs have neomorphic crania when compared with wolves. None of the over one hundred breeds represented in this study resembles either juvenile wolves or adult wolves; therefore, dogs could
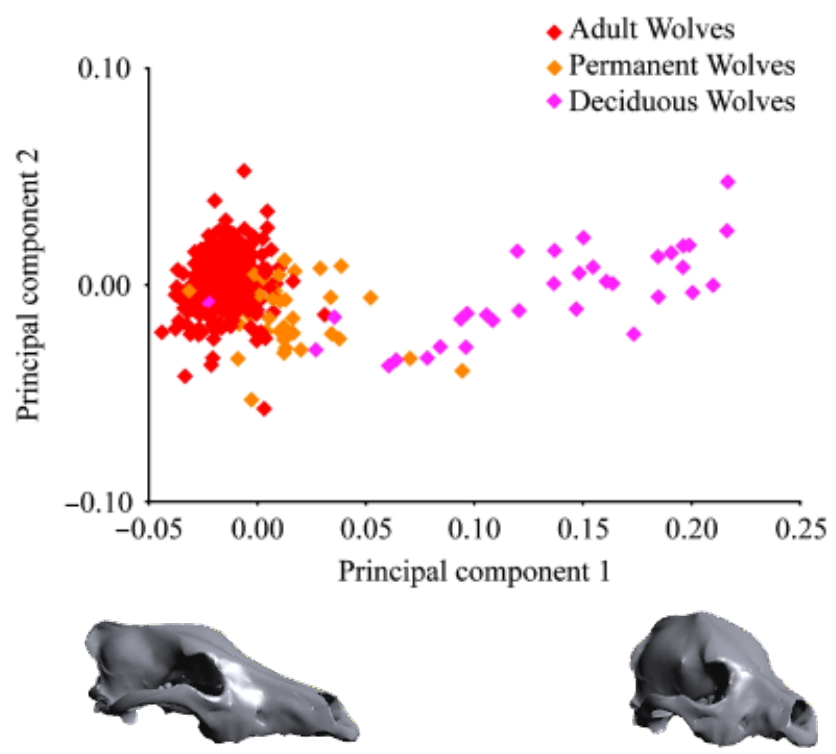

Fig. 4. Principal component analysis of the cranial shape variation in the ontogenetic series of wolves illustrating development of the wolf skull. This figure is available online in an interactive 3D format that requires Adobe Reader 9 or higher to view. not have evolved by simple truncation or retardation along the wolf cranial development trajectory. Modern dogs have a de novo rearrangement of the skull in which the palate and the basicranium rotate relative to each other. The muzzle is tilted dorsally in brachycephalic and mesaticephalic dogs (airorhynchy) or ventrally in down-face and dolichocephalic breeds (klinorhynchy). This pattern of variation between klinorhynchy and airorhynchy has been recognized before (Nussbaumer 1982; Fondon and Garner 2004; Drake and Klingenberg 2010). The pronounced angle between the nasal/ maxilla bones and the frontal bones, called a "stop," is present in mesaticephalic and brachycephalic breeds. Conversely, the wolf palate remains on the same plane with the basicranium throughout ontogeny and there is no "stop" present at the proximal end of the muzzle at birth or later in ontogeny. However, a small $(N=38)$ modern population of adult Dinaric-Balkan wolves was recently observed that have elevated muzzles and some cranial flexion (Milenković et al. 2010). The cranial flexion observed in this population of wolves may be due in part to the relative modularity of the face and neurocranium in wolves (Drake and Klingenberg 2010).

The modular nature of the face and neurocranium has been demonstrated for dogs, wolves, and even Carnivora and was shown to have a developmental basis (Drake and Klingenberg 2010). Gould (2002) suggested that heterochrony may occur in parts of organisms that are relatively independent from other parts resulting in a mosaic phenotype. However, neither of the separate investigations for regional heterochrony in the face or neurocranium of dogs revealed any shape similarities with adult or juvenile wolves. While the face and neurocranium are phenotypically and developmentally modular, the diversity of dogs has not occurred via separate heterochronic changes in these regions. On the other hand, it is clear that this phenotypic and developmental modularity may have facilitated the evolution of the novel skull arrangements that we observe in dogs.

Heterochrony is an alluring explanation for rapid and relatively simple evolutionary change via small genetic alterations. We often do not know the amount of genetic divergence between the organisms that we are comparing. Dogs and wolves have more in common genetically than any species previously tested for heterochrony (Mitteroecker et al. 2004; Blanco and Godfrey 2005; Mitteroecker et al. 2005). However, the heterochronic model fails to explain the evolution of domestic dogs from wolves.

Some phenotypic variation in complex biological structures like the vertebrate skull may not be adequately quantified with traditional distance analyses. The comprehensive description and quantification of shape provided by the geometric morphometric toolkit illuminates true differences in morphology between wolves and modern dogs that were not previously discernable with traditional two-dimensional bivariate plots. It is increasingly becoming the consensus 
that, while heterochrony may be recognized for some traits on bivariate plots, it is rarely found when the diagnosis is made in a multivariate context (Mitteroecker et al. 2004; Blanco and Godfrey 2005; Mitteroecker et al. 2005). For example, Blanco and Godfrey (2005) found that ontogenetic scaling of male and female howler monkeys was easily recognized on bivariate plots however a multivariate morphometric analysis detected significant differences in shape.

Some breeds of dogs, as Wayne (1986) pointed out, have short and wide skulls, as do wolf puppies. In fact, many brachycephalic breeds have a width to length ratio that is even more extreme than that found in wolf puppies (Drake 2004). However, this criterion does not mean that the cranial development of these breeds has been truncated at an early wolf developmental stage or that their development has been retarded. Bulldogs and similar breeds are different from juvenile wolves in that they have an upward tilting of the palate and extreme shortening of the nasal bones relative to the palate that is not found in wolf puppies. In wolf puppies the palate and basicranium lie in the same plane and the nasal bones are not significantly shorter than the palate.

Dogs are a model species for understanding the genetic and developmental mechanisms that lead to morphological diversity. Through the relaxation of many of the selection pressures that organisms face in nature we have created a unique environment for dog diversification. For instance, pet dogs are no longer required to hunt and kill their own prey or masticate tough animal tissues. In addition, many of the health problems associated with extreme facial morphologies such as breathing difficulties in brachycephalic dogs with elongated soft palates are now corrected with surgery (Torrez and Hunt 2006). On the other hand, purebred dogs are selected for very specific morphologies as defined in the breed standards (American Kennel Club 2006). Many of the traits selected for are perhaps unique in the history of evolution, for instance the "pendulous lips which blend into an ample dewlap" specified in the AKC breed standard of the Neapolitan Mastiff (American Kennel Club 2006; Drake and Klingenberg 2008). This relaxation of natural selection pressures with a coincident increase in artificial selection for unique traits has led, in part, to the extreme diversification of dog skull shapes.

The present study only addresses the morphological divergence of modern dogs because fossil specimens are rare and distinguishing fossil dogs from sympatric wolves is difficult (Parker et al. 2010). Many of the modern breeds were established 150 years ago through selection from small populations of individuals as well as the hybridization of many existing types of dogs (American Kennel Club, 2006). Once established, breed clubs did not allow dogs from outside the breed to be registered, creating reproductively separated populations that have since been under selection for a variety of behavioral and morphological traits (Coppinger and Coppinger 2001; Drake and Klingenberg 2008). Belyaev and
Trut's extensive fox domestication experiment (Trut et al. 2004) has suggested that the initial stages of domestication, the transition from fearful and aggressive behaviors to care-soliciting behaviors, are correlated with paedomorphic changes in skull morphology. They note that the domesticated foxes have wider and shorter skulls and look more similar to juvenile foxes. Whether the initial population of domestic dogs resembled juvenile wolves has yet to be tested. It is possible that the lack of correspondence between modern dogs and juvenile wolves is due to the initial hybridization and subsequent sustained selection for particular traits in modern dogs.

Closer investigation of patterns of cranial flexion in dogs may reveal the developmental changes underlying their diversity. Undoubtedly the modular independence of the face and neurocranium both developmentally and phylogenetically has played a role in the evolution of cranial flexion in dogs. Breeders seem to have unknowingly exploited this modularity, in St. Bernard's it has been established that cranial shape variation, in this case a dorsal tilting of the palate relative to the basicranium, can respond strongly to selection (Drake and Klingenberg 2008). In addition, recent analysis by Fondon and Garner (2004) of the correlation between developmental genes and dog skull shape variation support the hypothesis that simple genetic changes (in this case tandem repeats) may be associated with different skull morphologies, especially airorhynchy and klinorhynchy.

Dogs provide a unique opportunity to test models like heterochrony that attempt to link genetic, developmental, and phenotypic diversification. In a relatively short period of time dogs have rapidly evolved into a very phenotypically diverse species with very little genetic change. Heterochrony predicts that small genetic alterations to the ancestral ontogeny can lead to rapid diversification. However, the genetic changes to dog cranial development have not led to dog phenotypes that correspond to those expected by the heterochronic model. Future investigations of the genetic and developmental mechanisms that have given rise to the immense morphological variation of dogs will facilitate our understanding of how microevolutionary processes can lead to macroevolutionary patterns of diversity.

\section{Acknowledgments}

I am indebted to C. Corsano, R. Coppinger, M. Coquerelle, L. R. Godfrey, C. P. Klingenberg, and M. Sutherland for comments and suggestions on this manuscript. Marc Nussbaumer graciously provided the photographs of the St. Bernard skulls for Fig. 1. I am grateful to the curators of the following collections for access to specimens:

Smithsonian Institution's National Museum of Natural History, Washington, DC, USA; the private collection of Bonnie Dazell; the University of Alaska Museum in Fairbanks, Alaska, USA; Museum of Vertebrate Zoology at the University of California in Berkeley, California, USA; the Natural History Museum, London, UK; Natural History Museum in Berne, Switzerland; the Powell-Cotton Museum, Birchington, UK; and the Oxford University Museum of Natural 
History, Oxford, UK. This research was supported by a grant from The Leverhulme Trust and the US Department of Education.

\section{REFERENCES}

Alberch, P., Gould, S. J., Oster, G. F., and Wake, D. B. 1979. Size and shape in ontogeny and phylogeny. Paleobiology 5: 296-317.

American Kennel Club. 2006. The Complete Dog Book. Ballatine Books, New York.

Blanco, M. B., and Godfrey, L. R. 2005. Craniofacial sexual dimorphism in Alouatta palliata, the mantled howling monkey. J. Zool. 270: 268-276.

Bolk, L. 1926. Das problem der menschwerdung. Gustav Fischer, Jena.

Bookstein, F. L. 1991. Morphometric Tools for Landmark Data: Geometry and Biology. Cambridge University Press, Cambridge, UK.

Clutton-Brock, J., Corbet, G. B., and Hills, M. 1976. A review of the family Canidae, with a classification by numerical methods. Bull. Br. Mus. Nat. Hist. Zool. 29: 117-199.

Coppinger, L., and Coppinger, R. P. 1982. Livestock-guarding dogs that wear sheep's clothing. Smithsonian 13: 64-73.

Coppinger, R. P., and Coppinger, L. 2001. Dogs: A Startling New Understanding of Canine Origin, Behavior and Evolution. Scribner, New York.

Coppinger, R. P., Glendinning, J., Torop, E., Matthay, C., Sutherland, M., and Smith, C. K. 1987. Degree of behavioral neoteny differentiates Canid polymorphs. Ethology 75: 89-108.

de Beer, G. R. 1930. Embryology and Evolution. Clarendon Press, Oxford.

Dechambre, E. 1949. La théorie de foetalisation et la formation des races de chiens et de porc. Mammalia 13: 129-137.

Drake, A. G. 2004. Evolution and development of the skull morphology of canids: an investigation of morphological integration and heterochrony. $\mathrm{PhD}$ Dissertation, University of Massachusetts Amherst.

Drake, A. G., and Klingenberg, C. P. 2010. Large-scale diversification of skull shape in domestic dogs: disparity and modularity. Am Nat 175 289-301.

Drake, A. G., and Klingenberg, C. P. 2008. The pace of morphological change: historical transformation of skull shape in St Bernard dogs. Proc. R. Soc. Lond. B Biol. Sci. 275: 71-76.

Dryden, I. L., and Mardia, K. V. 1998. Statistical Shape Analysis. Wiley, Chichester.

Fondon, J. W. III., and Garner, H. R. 2004. Molecular origins of rapid and continuous morphological evolution. Proc. Natl. Acad. Sci. USA 101: 18058-18063.

Fox, M. W. 1965. Canine Behavior. Charles C. Thomas, Springfield.

Fox, M. W. 1978. The Dog: Its Domestication and Behavior. Garland STPM Press, New York.

Frank, H., and Frank, M. G. 1982. On the effects of domestication on canine social development and behavior. Appl. Anim. Ethol. 8: $507-525$.

Godfrey, L. R., King, S. J., and Sutherland, M. R. 1998. Heterochronic approaches to the study of locomotion. In E. Strasser, J. Fleagle, A. Rosenberger, and H. M. McHenry (eds.). Primate Locomotion: Recent Advances. Plenum Press, New York, pp. 277-307.

Good, P. 2000. Permutation Tests: A Practical Guide to Resampling Methods for Testing Hypotheses. 2nd Ed. Springer, New York.

Gould, S. J. 1977. Ontogeny and Phylogeny. The Belknap Press of Harvard University Press, Cambridge.

Gould, S. J. 2002. The Structure of Evolutionary Theory. Harvard University Press, Cambridge, MA

Howard, W. E. 1949. A means to distinguish skull of coyotes and domestic dogs. J. Mammal. 30: 169-171.

Klingenberg, C. P. 1998. Heterochrony and allometry: the analysis of evolutionary change in ontogeny. Biol. Rev. 73: 79-123.

Klingenberg, C. P. 2011. MorphoJ: an integrated software package for geometric morphometrics. Mol. Ecol. Res., advance online. DOI: 10.1111/j.1755-0998.2010.02924.x
Klingenberg, C. P., Barluenga, M., and Meyer, A. 2002. Shape analysis of symmetric structures: quantifying variation among individuals and asymmetry. Evolution 56: 1909-1920.

Klingenberg, C. P., and Monteiro, L. R. 2005. Distances and directions in multidimensional shape spaces: implications for morphometric applications. Syst. Biol. 54: 678-688.

Lawrence, B., and Bossert, W. H. 1969. The cranial evidence for hybridization in New England Canis. Mus. Comp. Zool. Harv. Univ. Brevoria 330: $1-13$.

McKinney, M. L. 1999. Heterochrony: beyond words. Paleobiology 25: 149-153.

Milenkovi, M., Šipetić, V. J., Blagojević, J., Tatović, S., and Vujošević, M. 2010. Skull variation in Dinaric-Balkan and Carpathian gray wolf populations revealed by geometric morphometric approaches. J. Mammal. 91: 376-386.

Miller, M. 1964. Anatomy of the dog. Saunders, Philadelphia.

Mitteroecker, P., Gunz, P., Bernhard, M., Schaefer, K., and Bookstein, F. L. 2004. Comparison of cranial ontogenetic trajectories among hominoids. J. Hum. Evol. 46: 679-698.

Mitteroecker, P., Gunz, P., and Bookstein, F. L. 2005. Heterochrony and geometric morphometrics: a comparison of cranial growth in Pan paniscus versus Pan troglodytes. Evol. Dev. 7: 244-258.

Morey, D. F. 1992. Size, shape, and development in the evolution of the domestic dog. J. Archaeol. Sci. 19: 181-204.

Morey, D. F. 1994. The early evolution of the domestic dog. Am. Sci. 82: 336-347.

Nussbaumer, M. 1982. Über die Variabilität der dorso-basalen Schädelknickungen bei Haushunden. Zool Anz 209: 1-32.

Olsen, S. J., and Olsen, J. W. 1977. The Chinese wolf, ancestor of new world dogs. Science 197: 533-535.

Parker, H. G., Shearin, A. L., and Ostrander, E. A. 2010. Man's best friend becomes biology's best in show: genome analyses in the domestic dog. Аппи. Rev. Genet. 44: 309-336.

Price, E. O. 1984. Behavioral aspects of animal domestication. Q. Rev. Biol. 59: $1-32$.

Raff, R. A., and Wray, G. A. 1989. Heterochrony: developmental mechanisms and evolutionary results. J. Evol. Biol. 2: 409-434.

Torrez, C. V., and Hunt, G. B. 2006. Results of surgical correction of abnormalities associated with brachycephalic airway obstruction syndrome in dogs in Australia. J. Small Anim. Pract. 47: 150-154.

Trut, L. N., Plyusnina, I. Z., and Oskina, I. N. 2004. An experiment on fox domestication and debatable issues of evolution of the dog. Russ. J. Genet. 40: 644-655.

Wayne, R. K. 1986. Cranial morphology of domestic and wild canids: the influence of development on morphological change. Evolution 40 243-261.

Wiley, D. F., et al 2005. Evolutionary morphing. Proceedings of the IEEE Visualization 2005 VIS '05, Minneapolis, pp. 431-438.

Zelditch, M. L., and Fink, W. L. 1996. Heterochrony and heterotopy: stability and innovation in the evolution of form. Paleobiology 22: 241-254.

Zeuner, F. E. 1963. A History of Domesticated Animals. Harper and Row, New York.

\section{SUPPORTING INFORMATION}

Additional supporting information may be found in the online version of this article:

\section{Table S1: Breed Sample Sizes.}

Please note: Wiley-Blackwell is not responsible for the content or functionality of any supporting materials supplied by the authors. Any queries (other than missing material) should be directed to the corresponding author for the article. 